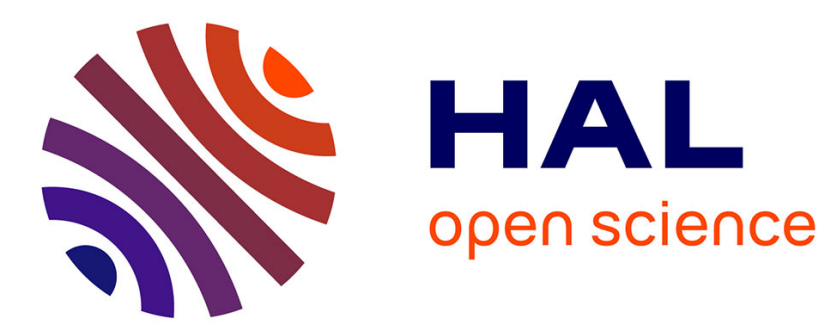

\title{
Influence et dialogisme
}

S. Laurens, Ivana Markova

\section{- To cite this version:}

S. Laurens, Ivana Markova. Influence et dialogisme. Bulletin de psychologie, 2011, Numéro 515 (5), pp.387 - 390. 10.3917/bupsy.515.0387 . hal-01780072

\section{HAL Id: hal-01780072 \\ https://hal.univ-rennes2.fr/hal-01780072}

Submitted on 27 Apr 2018

HAL is a multi-disciplinary open access archive for the deposit and dissemination of scientific research documents, whether they are published or not. The documents may come from teaching and research institutions in France or abroad, or from public or private research centers.
L'archive ouverte pluridisciplinaire HAL, est destinée au dépôt et à la diffusion de documents scientifiques de niveau recherche, publiés ou non, émanant des établissements d'enseignement et de recherche français ou étrangers, des laboratoires publics ou privés. 


\section{INFLUENCE ET DIALOGISME}

Stéphane Laurens, Ivana Marková

Groupe d'études de psychologie | «Bulletin de psychologie »

2011/5 Numéro 515 | pages 387 à 390

ISSN 0007-4403

Article disponible en ligne à l'adresse :

https://www.cairn.info/revue-bulletin-de-psychologie-2011-5-page-387.htm

\section{Pour citer cet article :}

Stéphane Laurens, Ivana Marková« Influence et dialogisme », Bulletin de psychologie 2011/5 (Numéro 515), p. 387-390.

DOI 10.3917/bupsy.515.0387

Distribution électronique Cairn.info pour Groupe d'études de psychologie.

(c) Groupe d'études de psychologie. Tous droits réservés pour tous pays.

La reproduction ou représentation de cet article, notamment par photocopie, n'est autorisée que dans les limites des conditions générales d'utilisation du site ou, le cas échéant, des conditions générales de la licence souscrite par votre établissement. Toute autre reproduction ou représentation, en tout ou partie, sous quelque forme et de quelque manière que ce soit, est interdite sauf accord préalable et écrit de l'éditeur, en dehors des cas prévus par la législation en vigueur en France. Il est précisé que son stockage dans une base de données est également interdit. 


\section{LAURENS Stéphane* MARKOVÁ Ivana** \\ Influence et dialogisme}

Les théories de l'influence sociale peuvent aisément illustrer la thèse selon laquelle les concepts ne sont pas des outils neutres permettant de décrypter objectivement des phénomènes préexistants et indépendants de la lecture qu'on en fait. Les concepts, utilisés dans ces théories, semblent être des éléments constitutifs de l'objet, ils participent à sa construction plus qu'ils en donnent une lecture ou ne l'expliquent.

Par exemple, l'usage des oppositions structurales, telles que « source - cible » ou « émetteur récepteur », pose, d'emblée, que le rapport d'influence est asymétrique : l'influence va de la source ou de l'émetteur vers la cible ou le récepteur. Ces concepts imposent l'idée selon laquelle le rapport d'influence est une emprise, une domination exercée par certains (les sources) sur d'autres (les cibles). La littérature expérimentale, loin d'infirmer ce schéma de base, l'a, plutôt, enrichi et coloré, en présentant des sources caractérisées par leur crédibilité, leur autorité, leur pouvoir, leur prestige, leur attractivité, leur supériorité numérique..., tandis que la cible était typiquement un sujet naïf, récepteur passif des influences. Ainsi, non seulement ces couples de concepts, au centre des théories de l'influence, imposent l'évidence d'une relation asymétrique, mais ils précisent, aussi, les statuts différenciés et complémentaires des entités en présence : la source possède des ressources psychologiques et la cible en est dépourvue (voir l'analyse critique de Moscovici, 1976/1979).

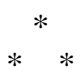

Cette édification conceptuelle, véritable construction d'une réalité souveraine, donnant à voir et à penser le monde (Berger, Luckman, 1966/1996), impose, aussi, une conception individualiste : l'influence ou l'interaction sont utilisées pour analyser comment deux entités indépendantes (sujet, autrui, groupe, institution, etc.), des unités séparées les unes des autres, agissent les unes sur les autres (Marková, 2003/2005). C'est bien à cette indépendance d'entités indivisibles et généralement fermées les unes aux autres, que répond la notion d'influence en psychologie sociale. On y observe, donc, comment l'individu (indivis), habituellement caractérisé par son autonomie de perception et de jugement, devient, par l'influence d'autrui, un assujetti (subjectum). Les fameuses expériences d'Asch ou de Milgram illustrent ce changement.

Dans cette logique, la société semble constituée d'individus autonomes et rationnels, percevant et analysant le monde aussi correctement que possible. Ce rapport direct de chacun au monde pose l'influence comme un phénomène fondamentalement perturbateur: ces perceptions et jugements seraient troublés par l'influence, faussée par l'intervention des autres (Dupuy, 1991). L'emprise ou la tentative d'emprise d'autrui sur l'individu lui ferait perdre son autonomie, ses moyens. Perdant son autonomie, se laissant influencer par autrui, sa nature même est modifiée. Il est transformé en énergumène, possédé, automate, agent... Or, la psychologie de cet individu sous emprise est un véritable mystère, qui n'est jamais été véritablement éclairé par les multiples ébauches de conceptualisations dont il a fait l'objet.

D'un autre côté, cette conception de l'influence pose autrui comme un danger potentiel pour le sujet: il est celui qui menace la validité de ses perceptions et de ses jugements et celui qui l'anéantit (par son influence), en tant qu'entité indépendante et autonome. La croyance en l'existence d'un puissant, et, donc, dangereux, instrument d'emprise, conduit à un sentiment d'insécurité, à une fermeture des individus et de la société, et incite à une désignation et une dénonciation de groupes, auxquels sont attribués ces intentions

\footnotetext{
* Centre de recherches en psychologie, cognition et communication, CRPCC, EA1285, Université Rennes 2, France.

** Université de Stirling, Écosse.

Correspondance: Stéphane Laurens, Université Rennes 2, Place du recteur Henri Le Moal, CS 24307 , 35043 Rennes cedex, France. <stephane.laurens@uhb.fr>
} 
néfastes. De tels mécanismes, qui se reproduisent régulièrement, provoquent des attitudes de discrimination et favorisent l'instauration de politiques liberticides : l'âge d'or de l'hypnose fut marqué par des débats à propos de ses dangers (si la connaissance des techniques d'induction hypnotique sortait du milieu médical pour être utilisée par chacun, ces derniers se serviraient, notamment, de l'hypnose pour assouvir leurs plus bas instincts, d'où une volonté d'interdire les spectacles publics), de même que la dernière décennie fut marquée par des débats à l'Assemblée nationale et au Sénat à propos de la manipulation mentale. Ces débats, partant de la présupposition que l'influence est un instrument puissant et à la portée de tous, permettant l'emprise de l'un sur l'autre, mènent au contrôle et à la restriction de son utilisation : enlever son usage à ceux dont la société ne pourrait garantir les qualités (morales, institutionnelles...), pour la réserver à des sources valides (par exemple, les médecins) (Laurens, 2005). En substance, et ceci paraît à peine croyable dans une société démocratique, ces débats conduisent à définir la liste de ceux qui ont le droit, la légitimité pour exercer une influence et ceux qui ne l'ont pas!

* *

À l'instar de la critique que fit Claude LeviStrauss (1950), à propos de la théorie du don, de Marcel Mauss, il faut nous demander si ces théories de l'influence ne sont pas une croyance, une théorie naïve par laquelle deux indivis en opposition structurale (par exemple, source-cible) auraient besoin d'être reliés par une force d'influence. Le hau de Mauss et des aborigènes n'est-il pas, par analogie, l'influence de nos théoriciens ? Cette force extraordinaire d'influence, que chaque époque redécouvre, renomme, étudie... - possession, magnétisme, hypnose, suggestion, influence, manipulation-, sans jamais aboutir à une théorie valide (ni même des faits stables!), ne serait, peut-être, que «le produit de la réflexion indigène », reprise par les scientifiques, pour faire le lien, la synthèse, entre les entités, les indivis, préalablement séparés par la théorie.

C'est cette thèse que soutint Pierre Janet : l'acte social (comme commandement et obéissance) est divisé en parties par des opérations de répartitions, mais ces opérations de répartition d'un acte entier (consistant, par exemple, à distinguer celui qui a l'initiative de l'acte et celui qui l'exécute) sont des opérations d'interprétation, qui n'expliquent rien. Les faits montrent, au contraire, l'unité de l'acte et la confusion entre ce que fait le sujet et ce que fait l'autre: celui qui ordonne commence souvent l'exécution et celui qui exécute répète souvent en lui-même l'ordre; ainsi, chacun fait ce que fait l'autre. De plus, chacun peut s'attribuer l'action entière (celui qui a ordonné et celui qui l'a exécutée). «Tous les actes sociaux sont du même genre, ils contiennent toujours une combinaison des actes du sujet et de ceux du socius plus ou moins confondus sur le même plan. Cette confusion, qui se trouve latente au fond de toute action sociale, est d'ordinaire dissimulée par les opérations de répartition »(Janet, 1937, p. 174).

Avec une telle lecture, la notion d'influence apparaît comme un élément des opérations de répartition, c'est-à-dire d'interprétation, mais elle n'explique pas l'acte.

L'objectif de ce dossier est de revenir sur certains présupposés des théories de l'influence et d'explorer de nouvelles voies, notamment en montrant tout l'intérêt d'autres interprétations de ces phénomènes. Ces nouvelles voies, abordées ici, ont deux points communs : le rejet du « dogme de l'unité indivisible et absolue de l'être humain » (Durand de Gros, 1867, p. 603) et, au contraire, l'affirmation que le sujet et autrui s'interpénètrent. L'autre n'est pas extérieur, il constitue le sujet, comme le sujet le constitue.

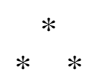

La plupart des théories de psychologie sociale conçoivent l'influence comme un flux d'information de la source au récepteur. Ce modèle monologique de communication est fondé sur la présupposition théorique d'un flux d'information à sens unique. Par contraste, dans ce dossier, nous porterons l'attention sur le modèle dialogique de l'influence.

Le modèle monologique de communication, qui a dominé la psychologie sociale, n'a, toutefois, pas été le seul dans ce domaine. Depuis la fin des années soixante, le processus d'influence et de communication a été étudié comme un processus de type dialogique, plutôt que monologique. La conception dialogique de l'influence prend sa source dans la recherche sur les interactions et les influences réciproques entre minorité et majorité (Moscovici, 1976/1979). Épistémologiquement, cette perspective fait dépendre l'influence d'une interdépendance entre l'Ego et l'Alter, qui agissent mutuellement l'un sur l'autre. Moscovici (Moscovici, Marková, 2000, p. 276) explique que la théorie de l'innovation des minorités, qu'il a développée, est, pour lui, un approfondissement de la théorie de la communication. Il précise : « Nombre de psychologues que je connais distinguent le phénomène de la communication du phénomène de l'influence [...]. Ma théorie est une théorie de l'influence: mais, en même temps, c'est une théorie du processus communicatif qui existe 
normalement entre les partisans et les opposants de points de vue différents » (Moscovici, Marková, 2000, p. 276).

La perspective dialogique (Marková, 2003/2005) se centre sur la capacité de l'esprit humain (l'Ego) à concevoir, à créer et à communiquer sur des réalités sociales en termes d'altérité (l'Alter), cela incluant d'autres personnes, idées ou symboles. L'Ego et l'Alter ne peuvent exister indépendamment comme deux éléments distincts, ils sont indissociablement articulés : ils se transforment mutuellement dans et à travers la communication et la pensée sociale.

Sur le plan de la psychologie sociale, l'interdépendance entre l'Ego et l'Alter se manifeste de deux façons opposées, mais complémentaires. D'une part, l'Ego et l'Alter veulent appartenir l'un à l'autre, s'atteindre l'un l'autre et partager des expériences de manière intersubjective. D'un autre côté, l'Ego et l'Alter ressentent le désir d'être mutuellement reconnus comme sujets. Ici, encore, la psychologie sociale a exploré la recherche de la reconnaissance sociale, à travers les études sur l'influence sociale (Moscovici, 1976/1979). Ces deux aspects de l'interaction Ego-Alter se manifestent, par exemple, comme une opposition dialogique entre conformité et innovation ou comme une contradiction résultant du désir d'identification de l'Ego et l'Alter, tout en revendiquent simultanément son autonomie. L'Ego et l'Alter s'influencent et se transforment mutuellement à travers ces procédés dialogiques complémentaires. Leur influence mutuelle peut se révéler non intentionnelle et s'exercer implicitement sans qu'ils s'en rendent compte; mais elle peut, tout aussi bien, être intentionnelle et explicite.

Le langage est intentionnel de différentes façons et recouvre, au moins, trois significations distinctes (Bühler, 1982). Tout d'abord, une personne exprime ce qu'elle a en tête, que ce soit un contenu, des idées, des sentiments, etc. Simultanément, elle s'adresse à quelqu'un, dont elle cherche à capter l'attention. Enfin, elle fait référence à certains objets ou contenus, au moyen de la parole.

À l'instar d'autres formes de la communication dialogique, l'influence peut impliquer différents types d'Ego et d'Alter, qui, dans des environnements sociaux précis, révèlent leurs particularités. Par exemple, l'influence peut intervenir, entre des individus, à un niveau interpersonnel; entre des individus et des groupes; entre des minorités et des majorités ; entre les médias et leur audience, etc. En outre, l'influence peut se manifester dans l'esprit même de l'Ego sous la forme d'un dialogue intérieur ; l'Ego en question peut, simultanément, être en train de mener un dialogue externe avec un Alter. Celui qui parle influence, à la fois, à travers le silence et la verbalisation, comme dans la rhétorique ou les interprétations authentiques ou bien à travers la duperie et le secret, ou encore en exprimant des significations directes ou indirectes. On peut influencer en flattant, en introduisant des nuances dans son message, on peut faire appel à un tiers, etc. En d'autres termes, comme toutes les formes de communication, l'influence est hétérogène.

La perspective dialogique, dans le cas d'une minorité se battant pour faire valoir ses idées, implique l'existence d'une relation d'interdépendance entre cette minorité et la majorité. Autrement dit, minorité et majorité se constituent ensemble, elles s'influencent l'une l'autre, réciproquement. Ces influences de la majorité et de la minorité reposent, cependant, sur des processus distincts: l'influence de la majorité conduit souvent à la conformité tandis que l'influence de la minorité peut produire de la créativité, de l'innovation. De même, si l'influence de la majorité s'observe dans des réponses manifestes (réponses publiques, directes, immédiates), l'individu adoptant la position majoritaire sans, pour autant, changer d'avis intimement, au niveau latent (réponses privées, indirectes, différées), les minorités, au contraire, ont rarement une influence manifeste, mais en obtiennent au niveau latent (la conversion).

Pour comprendre les processus de l'influence, il est nécessaire de les appréhender dans leur cadre, dans les systèmes auxquels ils appartiennent (par exemple, les institutions, les organisations, la communication), plutôt que de les étudier comme des phénomènes isolés. Ils englobent des processus de communication conscients et inconscients, ce « retour de l'inconscient» (Moscovici, 1993) qui, au début du $\mathrm{XX}^{\mathrm{e}}$ siècle, déconcerta la psychologie qui, dans sa volonté de faire science, ne savait comment rattacher l'inconscient à ses modèles de pensée rationnelle. Ce retour de l'inconscient, a conduit à abolir des distinctions comme celle entre phénomènes dits normaux et ceux dits anormaux, entre l'individuel et le collectif. Si la psychologie sociale a été troublée par la notion de pensée implicite et inconsciente, aujourd'hui encore, la majorité des expériences réalisées en psychologie sociale préconisent de s'en tenir à l'étude des phénomènes relatifs au niveau conscient. Dans la communication dialogique, cependant, la pensée latente et inconsciente et le dialogue intérieur sont considérés comme des aspects inévitables de la communication, qu'il s'agisse des processus d'influence ou des représentations sociales. Tous reposent et émanent de processus historico-culturels, et déterminent les significations symboliques de la communication sociale présente et à venir. 


\section{RÉFÉRENCES}

BERGER (Peter Ludwig), Luckmann (Thomas).- The social construction of reality : a treatise in the sociology of knowledge, New York, Doubleday, 1966 ; trad. fr., La construction sociale de la réalité, Paris, Armand Colin, 1996.

BÜHLER (Karl).- The axiomatization of the language sciences, dans Innis (R. E.), Karl Bühler. Semiotic foundations of language theory, New York et Londres, Plenum, 1982.

Dupuy (Jean Pierre).- La panique, Paris, Les empêcheurs de penser en rond, 1991.

DurAND DE GRos (Joseph-Pierre).- Polysoïsme ou pluralité animale dans l'homme, Bulletin de la société d'anthropologie de Paris, séance du 7 novembre, 1867, p. 600-627.

JANET (Pierre).- Les troubles de la personnalité sociale, Annales médico-psychologiques, 95, XV, 1937, p. $149-200$ et p. 421-468. Bulletin de psychologie, 414, 1994 , p. $156-183$.
LAURENS (Stéphane).- L'influence, entre science et fantasme, Hermès, 41, p. 83-90.

LÉVI-STRAUSS (Claude).- Introduction à l'œuvre de Marcel Mauss, dans Mauss (M.), Sociologie et anthropologie, Paris, Presses universitaires de France, 1950.

MARKovÁ (Ivana).- Dialogicity and social representation, Cambridge, Cambridge university press, 2003 ; trad. fr., Dialogicité et représentations sociales, Paris, Presses universitaires de France, 2005.

Moscovici (Serge).- Social influence and social change, Londres et New York, Academic press, 1976 ; trad. fr., Psychologie des minorités actives, Paris, Presses universitaires de France, 1979.

Moscovici (Serge).- The return of the unconscious, Social research, 60, 1993, p. 39-93.

Moscovici (Serge), MARKovÁ (Ivana).- Ideas and their development : a dialogue between S. Moscovici and I. Marková, dans Moscovici (S.), Social representations : explorations in social psychology, Cambridge, Polity press, 2000, p. 224-286. 